\title{
The Flexible On-Surface Self-Assembly of a Low-Symmetry Mabiq Ligand: An Unconventional Metal-Assisted Phase Transformation on $\mathrm{Ag}(\mathbf{1 1 1})$
}

Felix Haag ${ }^{\dagger}$, Peter S. Deimel ${ }^{\dagger}$, Peter Knecht ${ }^{\dagger}$, Lukas Niederegger ${ }^{\ddagger}$, Knud Seufert ${ }^{\dagger}$, Marc G. Cuxart $^{+}$, Yang $\mathrm{Bao}^{+}$, Anthoula C. Papageorgiou ${ }^{+}$, Matthias Muntwiler ${ }^{\S}$, Willi Auwärter ${ }^{\dagger}$, Corinna R. Hess ${ }^{\ddagger *}$, Johannes V. Barth ${ }^{\dagger}$ and Francesco Allegretti ${ }^{*}$ ${ }^{\dagger}$ Physics Department E20, Technical University of Munich, D-85748 Garching Germany ${ }^{\ddagger}$ Chemistry Department, Technical University of Munich, D-85748 Garching, Germany ${ }^{\S}$ Paul Scherrer Institut, $\mathrm{CH}-5232$ Villigen, Switzerland

*Email: corinna.hess@ch.tum.de; francesco.allegretti@ph.tum.de

\section{Supporting Information}

\section{Table of Contents}

1. TPD multilayer and monolayer supplementary data .................................................... S2

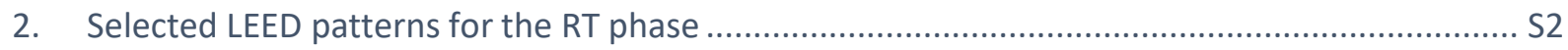

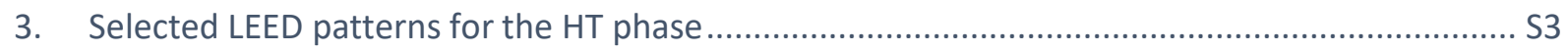

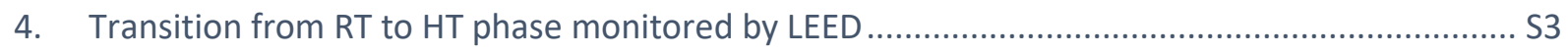

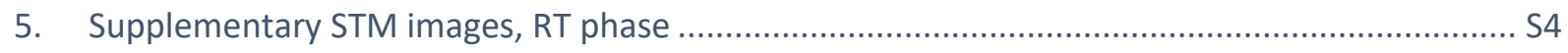

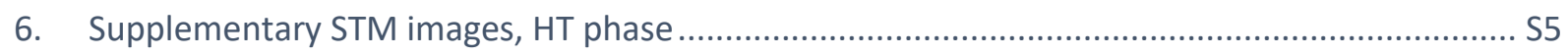

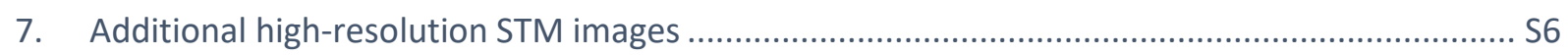

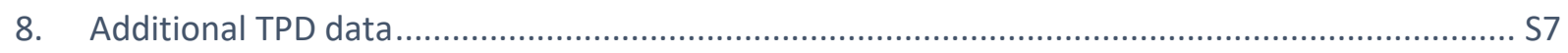

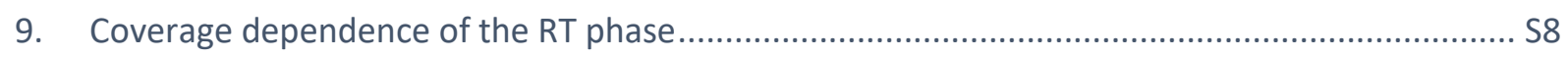

10. Observation of Ag-bridged trimetallic Mabiq dimers in solution ......................................... S9

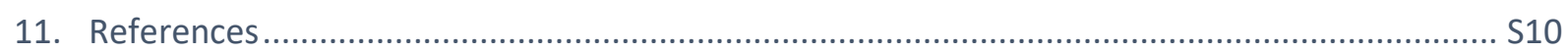




\section{TPD multilayer and monolayer supplementary data}
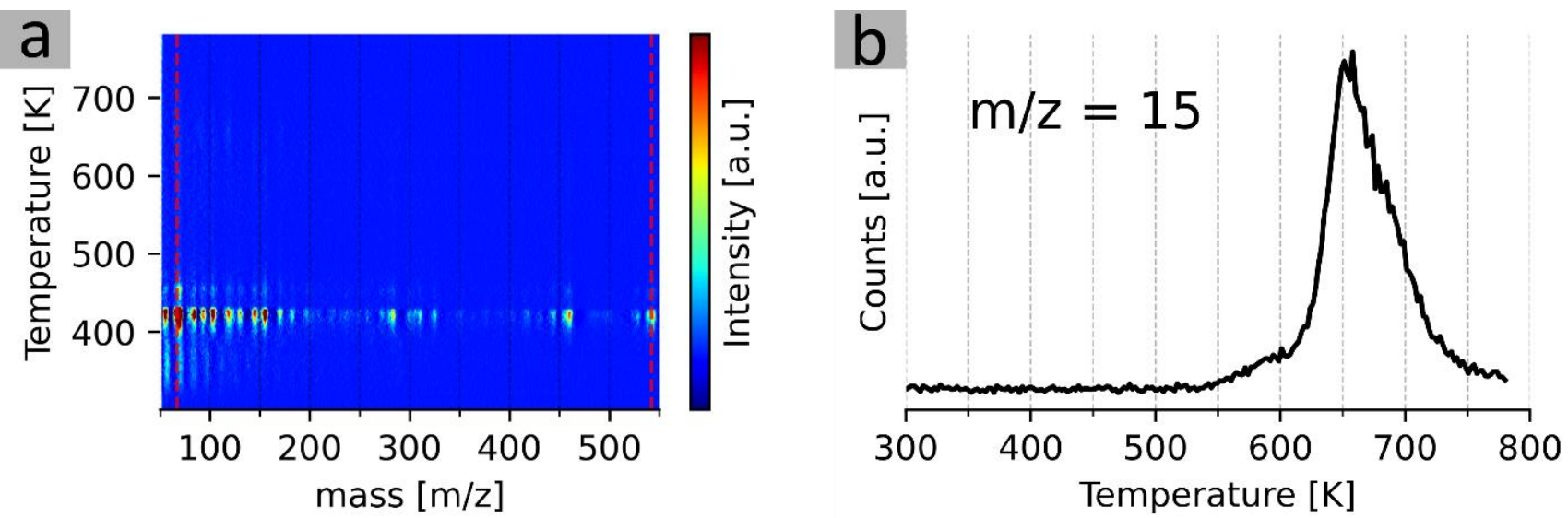

Figure S1: (a) Large-mass 2-D TPD spectrum ranging from $\mathrm{m} / \mathrm{z}=50$ to 550 for an $\mathrm{H}$-Mabiq multilayer with the main fragment $(\mathrm{m} / \mathrm{z}=67)$ and the parent ion $(\mathrm{m} / \mathrm{z}=542)$ indicated as red, dashed lines. Signal intensity is given in the color scale. (b) Thermally-induced decomposition of an H-Mabiq monolayer signaled by desorption of fragments with $\mathrm{m} / \mathrm{z}=15$, mainly associated to $\mathrm{CH}_{3}$ from terminal groups. The monolayer was prepared with the $\mathrm{Ag}(111)$ substrate held at $300 \mathrm{~K}$. Heating rate in $(a, b): 2 \mathrm{~K} / \mathrm{s}$.

\section{Selected LEED patterns for the RT phase}
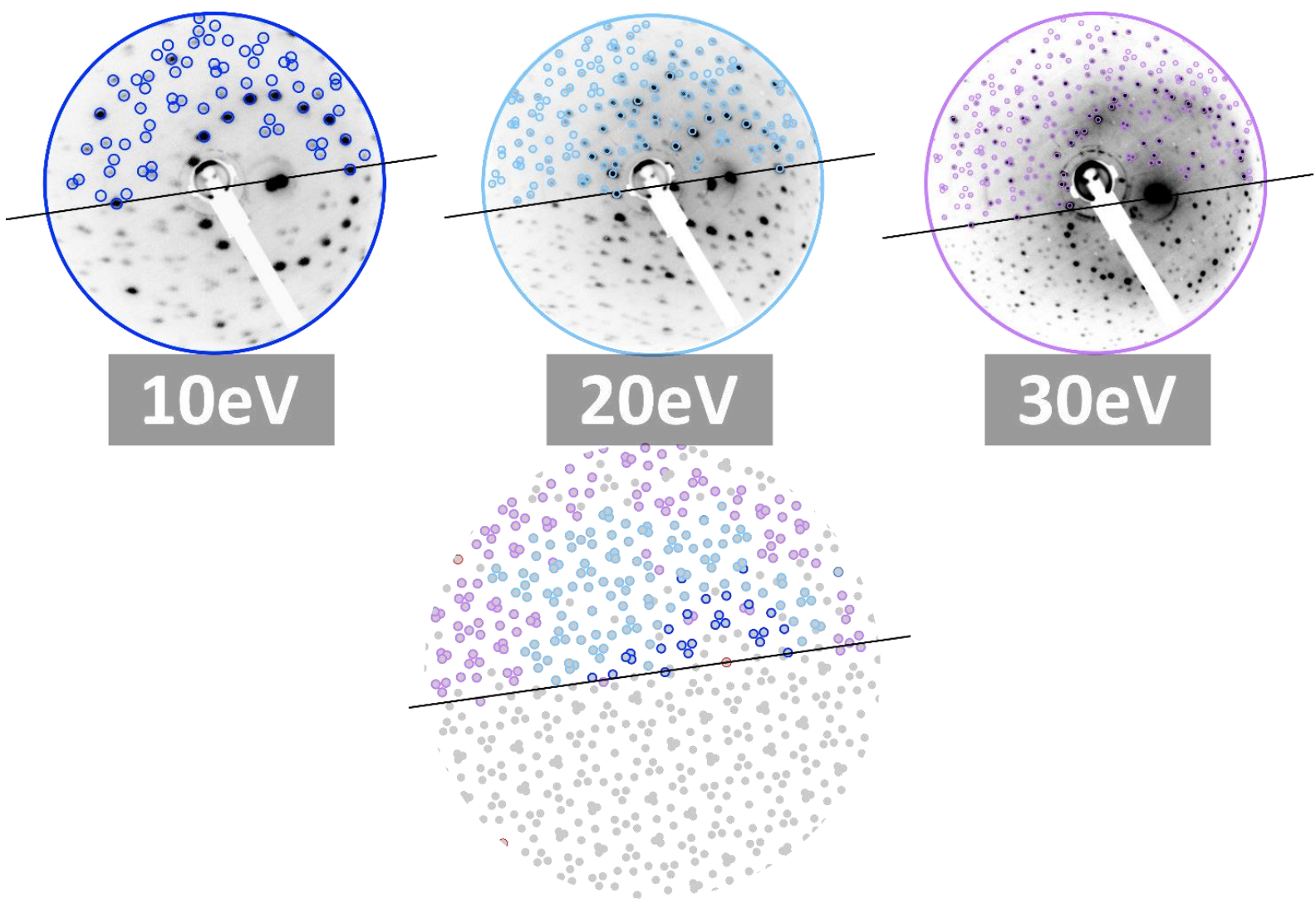

Figure S2: LEED patterns at three different primary electron energies (top) and simulated reciprocal-space pattern (bottom) for the H-Mabiq RT phase deposited with the substrate kept at room temperature (RT, $300 \mathrm{~K})$. Characteristic motifs observed are highlighted with circles of different colors in the LEED patterns and superimposed onto the calculated reciprocal-space pattern, which is based on a $\left(\begin{array}{cc}8 & 0 \\ -6 & 12\end{array}\right)$ superstructure matrix relative to the (111) substrate mesh. 


\section{Selected LEED patterns for the HT phase}

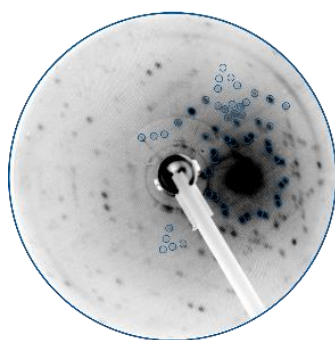

$15 \mathrm{eV}$

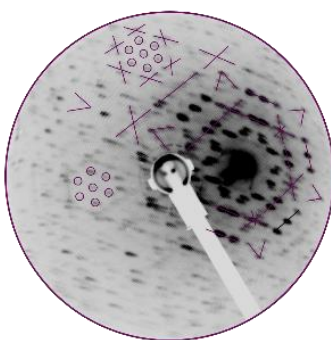

$20 \mathrm{eV}$

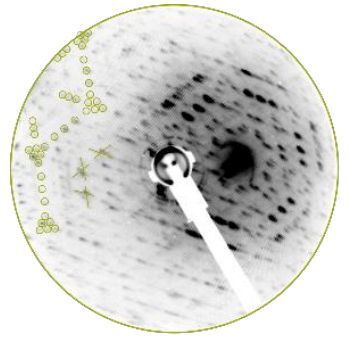

$25 \mathrm{eV}$

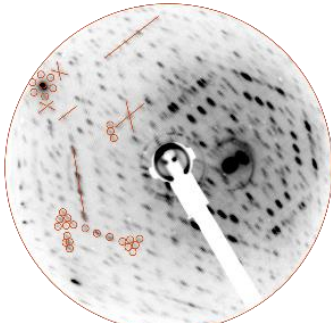

$30 \mathrm{eV}$

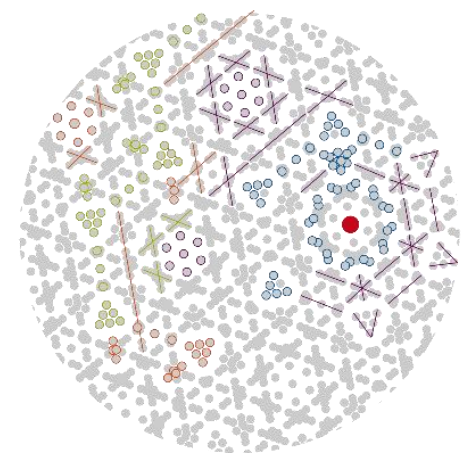

Figure S3: LEED patterns at various primary electron energies (top) and simulated reciprocal-space pattern (bottom) for the H-Mabiq HT phase deposited at high substrate temperature (HT, $500 \mathrm{~K})$. Each primary beam energy shown here reproduces a specific part of the overlayer superstructure particularly well, and corresponding motifs are highlighted with colored circles. The superstructure matrix for the reciprocal-space pattern was found to be $\left(\begin{array}{cc}9 & 0 \\ -5 & 16\end{array}\right)$ relative to the (111) substrate mesh.

\section{Transition from RT to HT phase monitored by LEED}

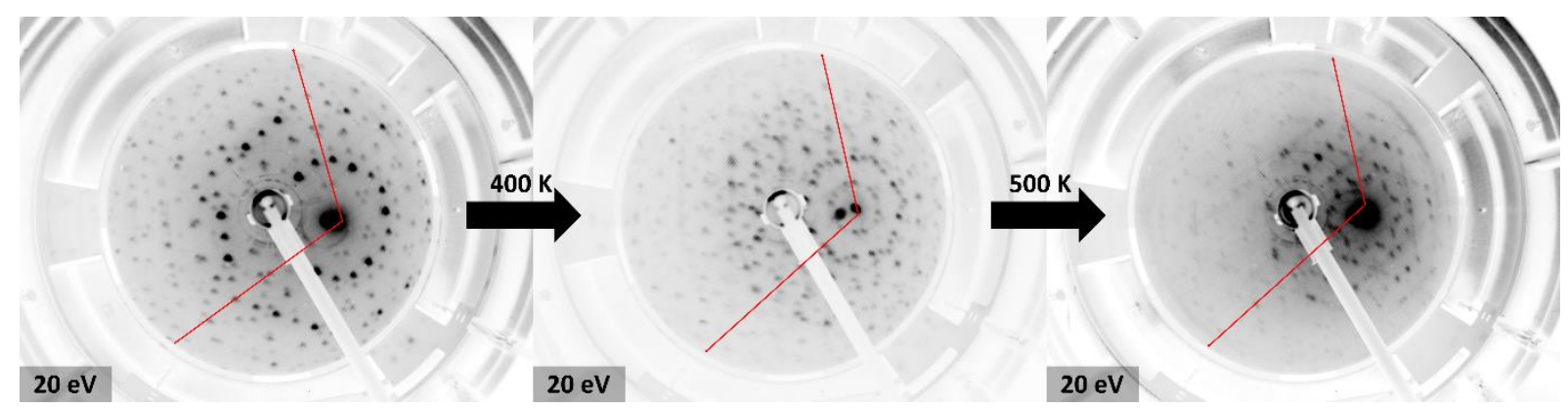

Figure S4: Temperature-dependent evolution of the H-Mabiq phase prepared at RT into the HT phase, monitored by LEED at a primary electron energy of $20 \mathrm{eV}$. The $300 \mathrm{~K}$ deposited system (left) shows a LEED pattern analogous to those of Figure $3 a$ in the main manuscript. The initial coverage of H-Mabiq was $0.8 \mathrm{ML}$ (determined by XPS, not shown). The H-Mabiq overlayer was annealed in steps at the indicated temperatures and then cooled down below $100 \mathrm{~K}$ for LEED acquisition. While the complex LEED pattern of the $400 \mathrm{~K}$ annealed overlayer contains elements of both the RT and HT phases (middle panel), at $500 \mathrm{~K}$ a largely pure HT phase is found (right panel). The red lines mark two of the three $<11 \overline{2}>$ symmetry directions of the Ag(111) substrate. 


\section{Supplementary STM images, RT phase}
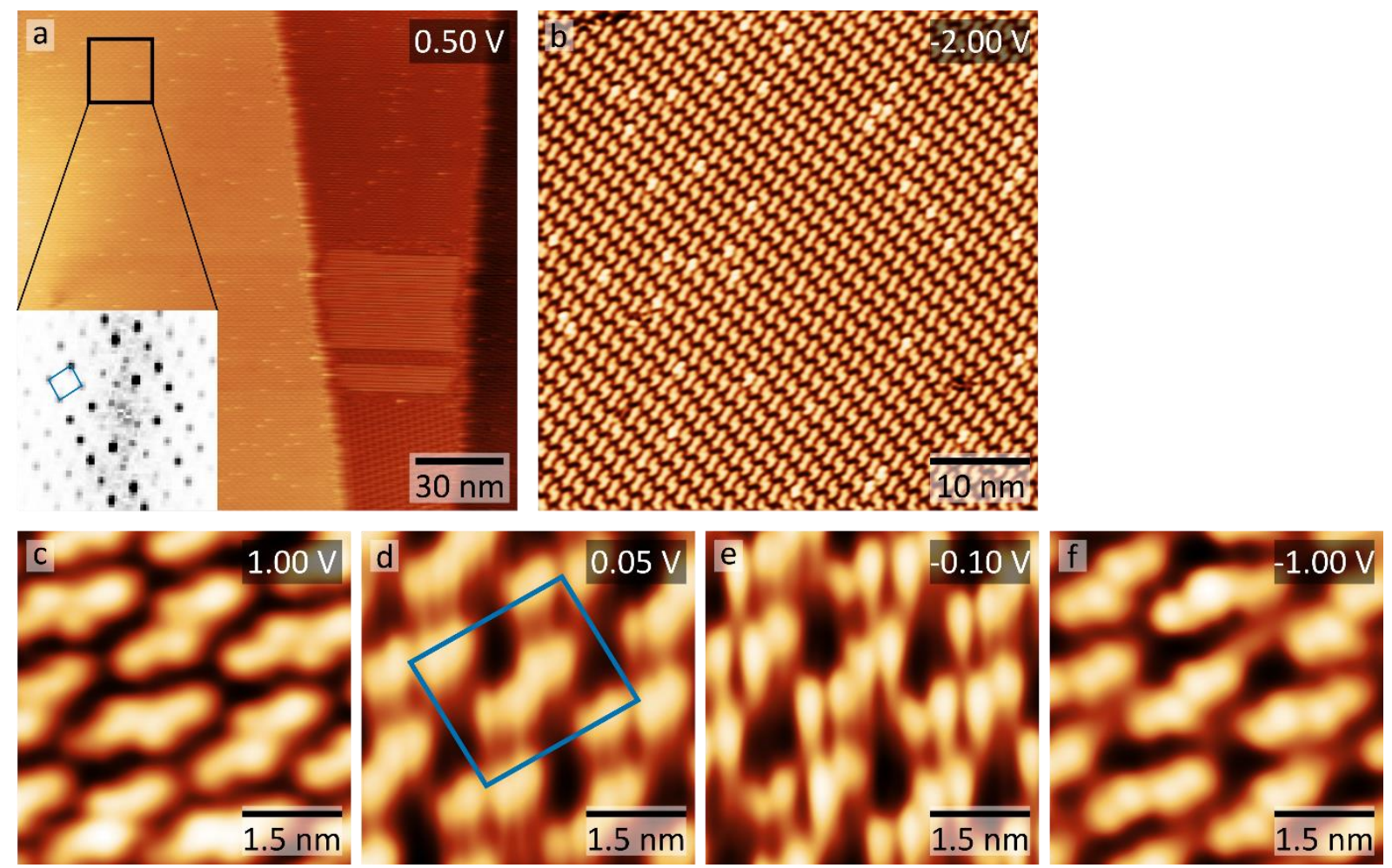

Figure S5: (a) Large-scale STM image of H-Mabiq/Ag(111), RT phase, at $4.2 \mathrm{~K}\left(\mathrm{U}_{B}=0.50 \mathrm{~V}, \mathrm{I}=0.10 \mathrm{nA} ; 170 \times 170\right.$ $\mathrm{nm}^{2}$ ), showing large terraces of well-ordered arrays of molecules at monolayer coverage. A domain of excess molecules on top of a monolayer island is also observed (middle). In the left-bottom corner, the 2-D Fast Fourier Transform (FFT) of the indicated area (black square) is shown as an inset. Gauging from the FFT, the unit cell vectors have dimensions of $2.4 \pm 0.2 \mathrm{~nm}$ and $2.9 \pm 0.2 \mathrm{~nm}$, respectively, with an angle of $91^{\circ}$ between them. (b) Zoom-in into an ordered molecular domain $\left(U_{B}=2.00 \mathrm{~V}, I=0.10 \mathrm{nA} ; 50 \times 50 \mathrm{~nm}^{2}\right)$. (c-f) $R T$ phase appearance at various bias voltages $\left(I=0.05 \mathrm{nA} ; 5.0 \times 5.0 \mathrm{~nm}^{2}\right)$ : (c) $U_{B}=1.00 \mathrm{~V}$, (d) $U_{B}=0.05 \mathrm{~V}$, (e) $U_{B}=-0.10 \mathrm{~V},(f) U_{B}=-1.00 \mathrm{~V}$. Note that the four images are not taken from exactly the same area of the sample. The primitive unit cell is drawn in (d). 


\section{Supplementary STM images, HT phase}
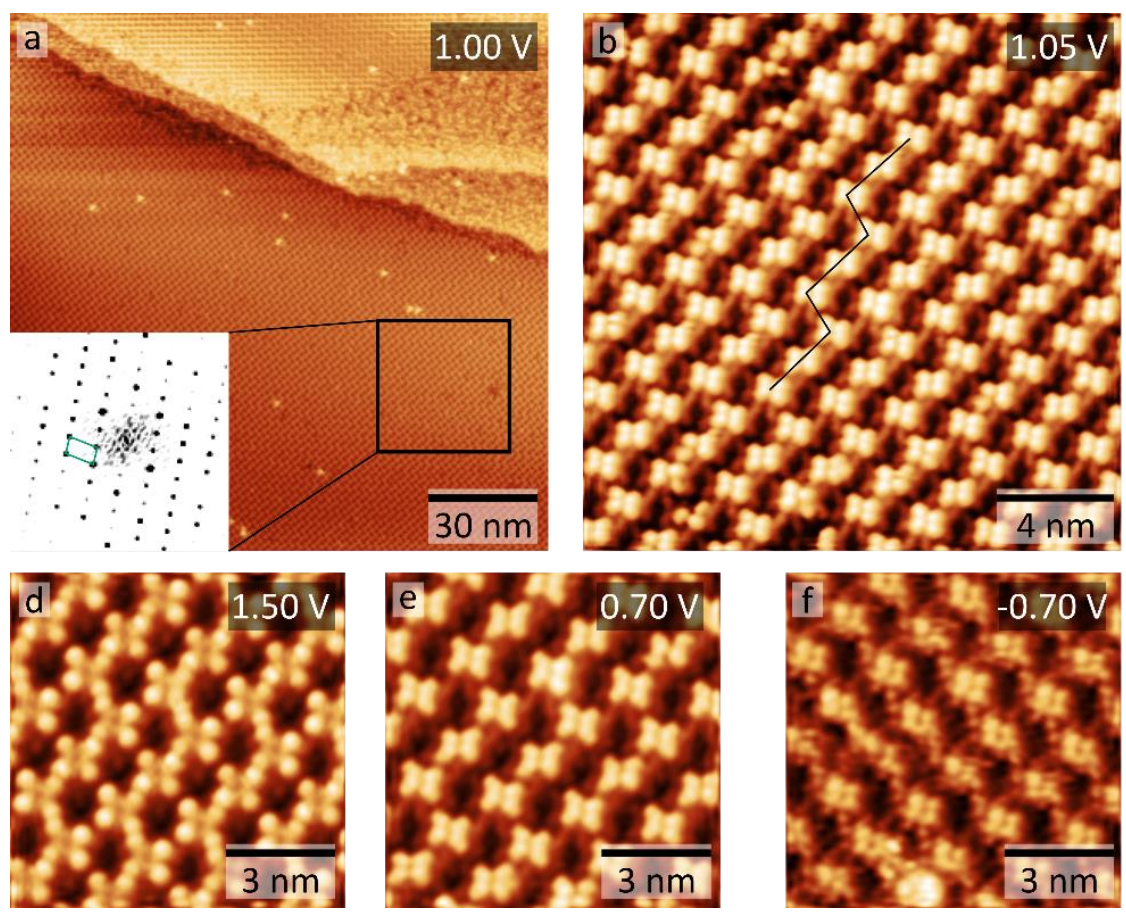

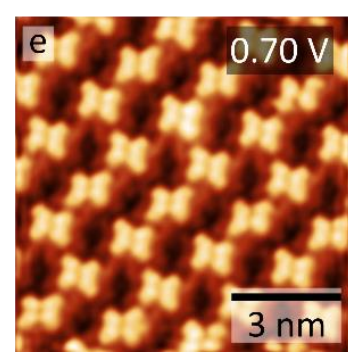

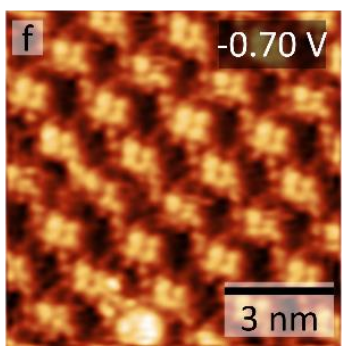
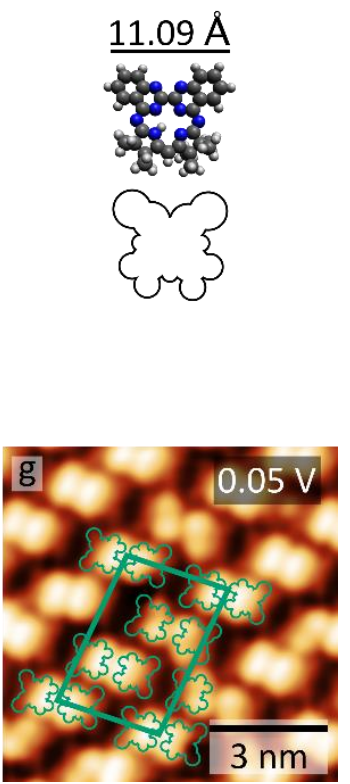

Figure S6: (a) Large-scale STM image of H-Mabiq/Ag(111), HT phase, at $6 \mathrm{~K}\left(\mathrm{U}_{B}=1 \mathrm{~V}, \mathrm{I}=0.13 \mathrm{nA} ; 148 \times 148 \mathrm{~nm}^{2}\right)$, showing a large, regular molecular domain (from top to bottom) along with a disordered island (top right part). The inset at the left-bottom corner displays the 2-D FFT of the area in the black square. From the FFT the unit cell dimensions are found to be $2.6 \pm 0.2 \times 4.2 \pm 0.2 \mathrm{~nm}^{2}$, with the two vectors enclosing an angle of $80^{\circ}$, which is in excellent agreement with the LEED-based analysis and the local real-space images (see main text). (b) Zoom-in into an ordered molecular domain $\left(U_{B}=1.05 \mathrm{~V}, I=0.13 \mathrm{nA} ; 18.4 \times 18.4 \mathrm{~nm}^{2}\right)$. The zig-zag motif which is evident in (a) is highlighted at this smaller scale by the black line. (c) Outline of the H-Mabiq molecule, derived from the molecular dimensions (cf. Figure $4 c$ in the main manuscript). (d-g) Bias-dependence of close-up topographic images with primitive unit cell and tentative model drawn in $(g)(S T M$ measurement parameters: $(d-f): I=0.13 n A$; $\left.9.2 \times 9.2 \mathrm{~nm}^{2} ;(g): I=0.20 \mathrm{nA} ; 8.0 \times 8.0 \mathrm{~nm}^{2} ;(d) U_{B}=1.50 \mathrm{~V},(e) U_{B}=0.70 \mathrm{~V},(f) U_{B}=-0.70 \mathrm{~V},(g) U_{B}=0.05 \mathrm{~V}\right)$. The molecular outline used in $(g)$ is the same as shown in (c). 


\section{Additional high-resolution STM images}

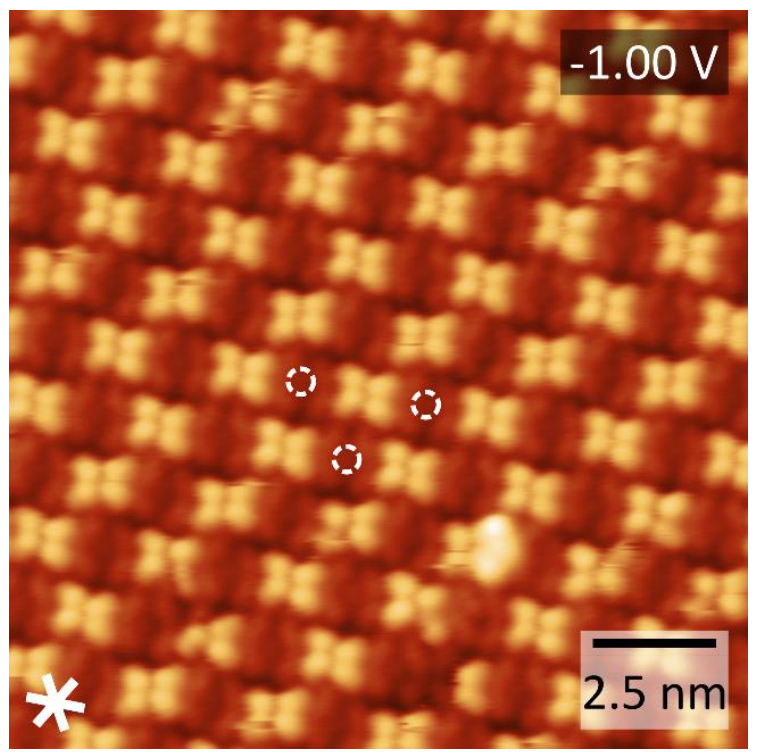

Figure S7: Medium-scale STM image of the H-Mabiq HT phase on $A g(111)$, taken at $6 \mathrm{~K}\left(U_{B}=-1.00 \mathrm{~V}, \mathrm{I}=0.13 \mathrm{nA}\right.$; $15 \times 15 \mathrm{~nm}^{2}$ ), showing an ordered molecular domain with regular intensity in-between H-Mabiq molecules as indicated by the white, dashed circles. The Ag(111) close-packed directions are indicated by white lines.

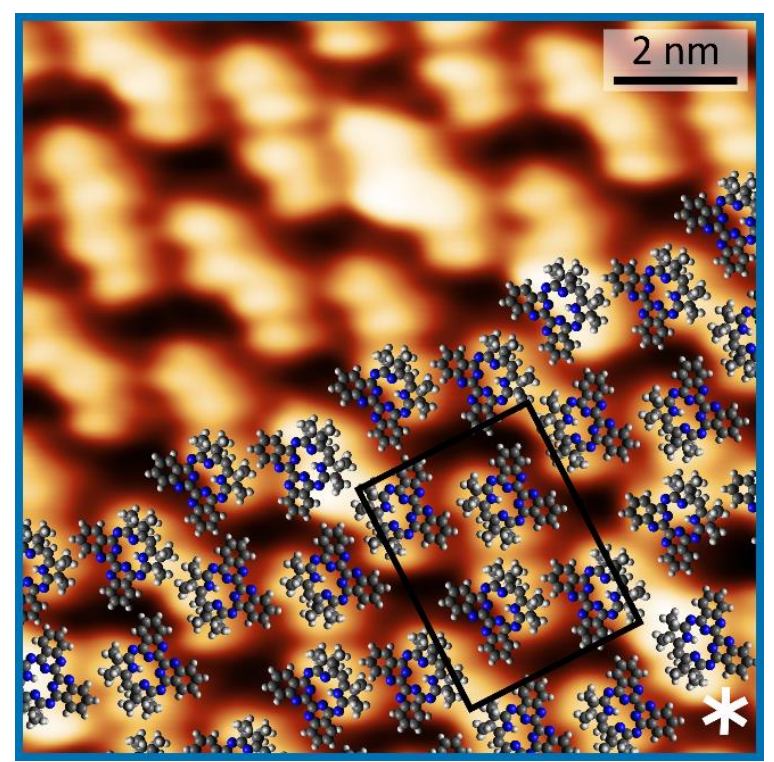

Figure S8: Ball-and-stick model overlaid on an ordered domain of RT deposited H-Mabiq, taken at 4.2 K. STM measurement parameters: $U_{B}=0.03 \mathrm{~V}, I=0.03 \mathrm{nA} ; 12 \times 12 \mathrm{~nm}^{2}$. The Ag(111) close-packed directions are indicated by the white lines. 


\section{Additional TPD data}

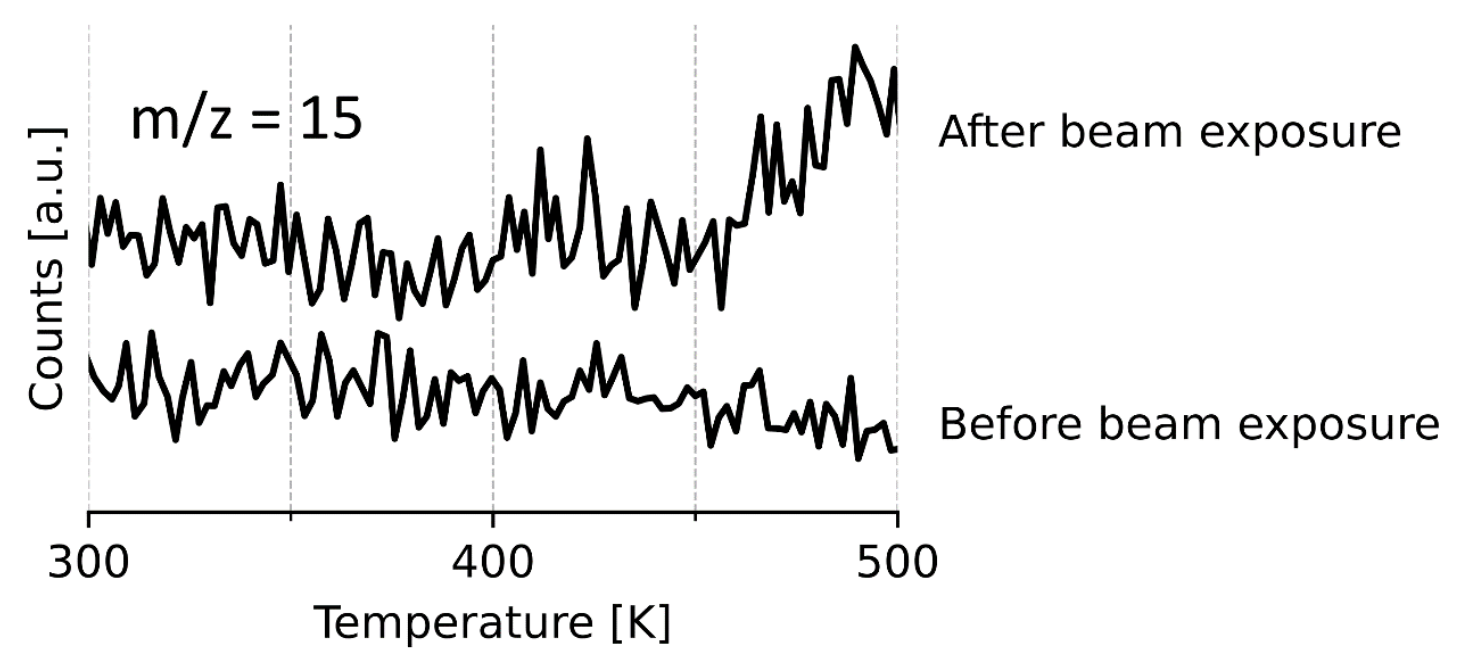

Figure S9: Comparison of $\mathrm{m} / \mathrm{z}=15$ desorption with and without radiation damage for an H-Mabiq monolayer prepared at $300 \mathrm{~K}$. (top) After prolonged beam exposure ( 30 min X-rays and 30 min electron beam) a weak desorption feature corresponding to $\mathrm{m} / \mathrm{z}=15$ is observed above $450 \mathrm{~K}$, most likely related to $\mathrm{C}_{\text {- }} \mathrm{CH}_{3}$ cleavage followed by desorption of the liberated methyl groups. Thus, it is argued that radiation damage causes a weakening of the $\mathrm{C}_{-} \mathrm{CH}_{3}$ bonds. (For the X-ray exposure, the $\mathrm{Mg}$ anode of the XPS setup was used, delivering Mg $K_{\alpha}$ radiation with photon energy $\hbar \omega=1253.6 \mathrm{eV}$ over the entire sample surface. Additionally, the sample was irradiated with a $30 \mathrm{eV}$ electron beam from the LEED electron gun. Since the beam diameter of the electron beam is relatively narrow ( $1 \mathrm{~mm}^{2}$ ), the sample position was changed in five-minute intervals.) (bottom) Without beam exposure, the molecule remains intact when heating to $500 \mathrm{~K}$, excluding the loss of $\mathrm{CH}_{3}$ groups as possible origin of the RT to HT phase transformation.

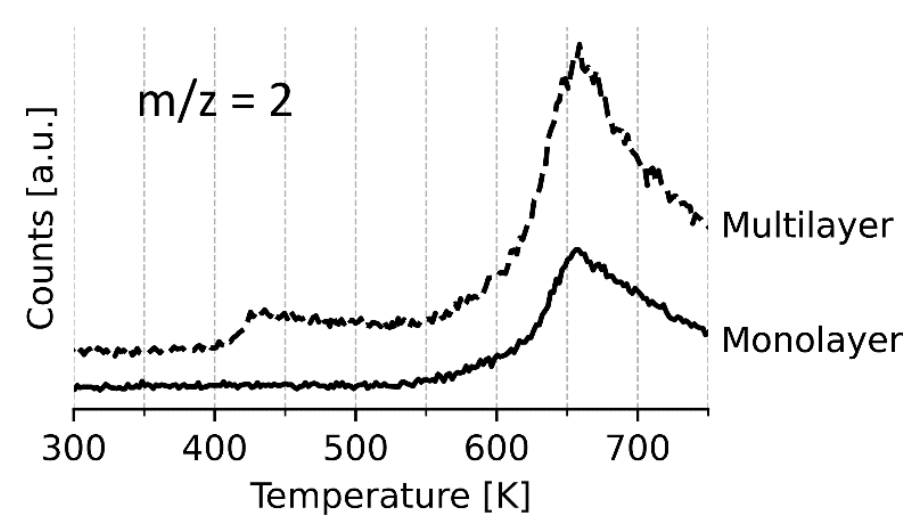

Figure S10: $\mathrm{H}_{2}$ TPD spectra for a monolayer and an ultra-thin multilayer of H-Mabiq deposited at $300 \mathrm{~K}$. The signal in the multilayer at $420 \mathrm{~K}$ is related to multilayer desorption and subsequent cracking in the mass spectrometer (cf. Figure 2 of the main manuscript). Starting from $600 \mathrm{~K}$ a strong signal related to $\mathrm{CH}_{3}$ release (see Figures S1b, S9) is observed for both coverages. Importantly, no hydrogen signal is detected for monolayer films upon annealing between 300 and $500 \mathrm{~K}$. Therefore, as also concluded by XPS (see main text), the RT to HT phase transformation is not associated to deprotonation of the aminic $N(-N H)$ species of the macrocycle. 


\section{Coverage dependence of the RT phase}
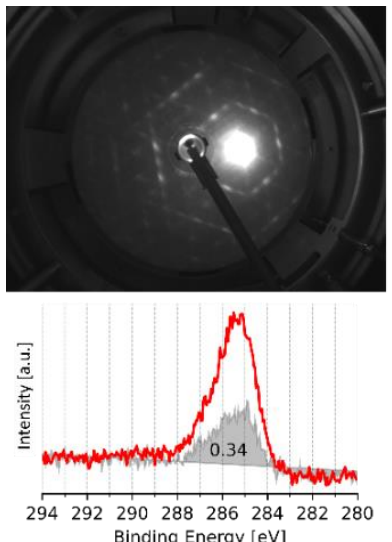
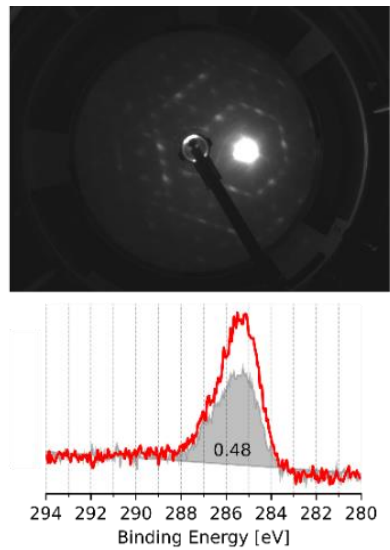
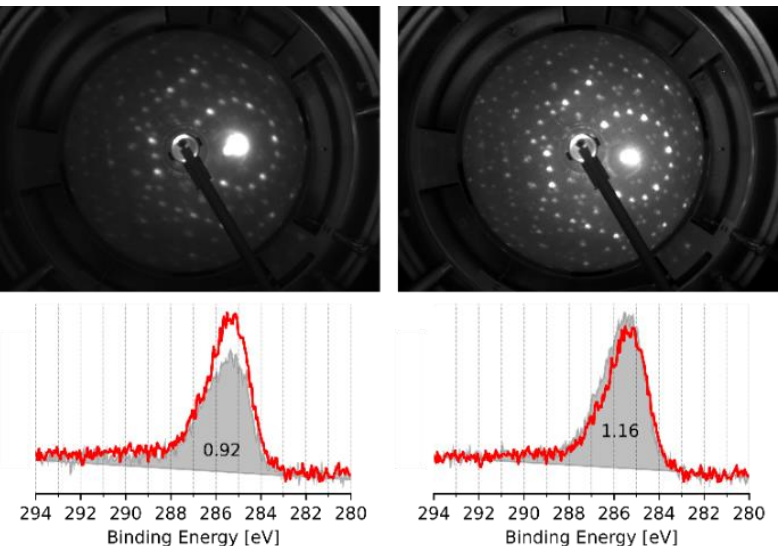

Figure S11: LEED images (top panels) for H-Mabiq deposited at RT on Ag(111) at different coverages up to (about) monolayer completion. All LEED images were taken at a primary electron energy of $20 \mathrm{eV}$. The coverage was determined by C 1s XPS (lower panels, data acquired with $\mathrm{Mg} \mathrm{K \alpha}$ radiation, $1253.6 \mathrm{eV}$ ) and referred to the full monolayer of the HT phase (saturation coverage at HT - red curve). From left to right the coverage thus varies between 0.34 and 1.16 of such a monolayer (note that with respect to the Ag(111) surface density, the HT phase corresponds to a relative coverage of about 0.04). Already at a coverage of $34 \%$ of the monolayer, a regular overlayer is formed, as signaled by the appearance of diffraction spots in LEED. With increasing coverage, the spots become progressively sharper and spot streaking disappears, indicating enhanced crystallinity. Notably, the characteristic RT phase ordering is observed at all coverages.
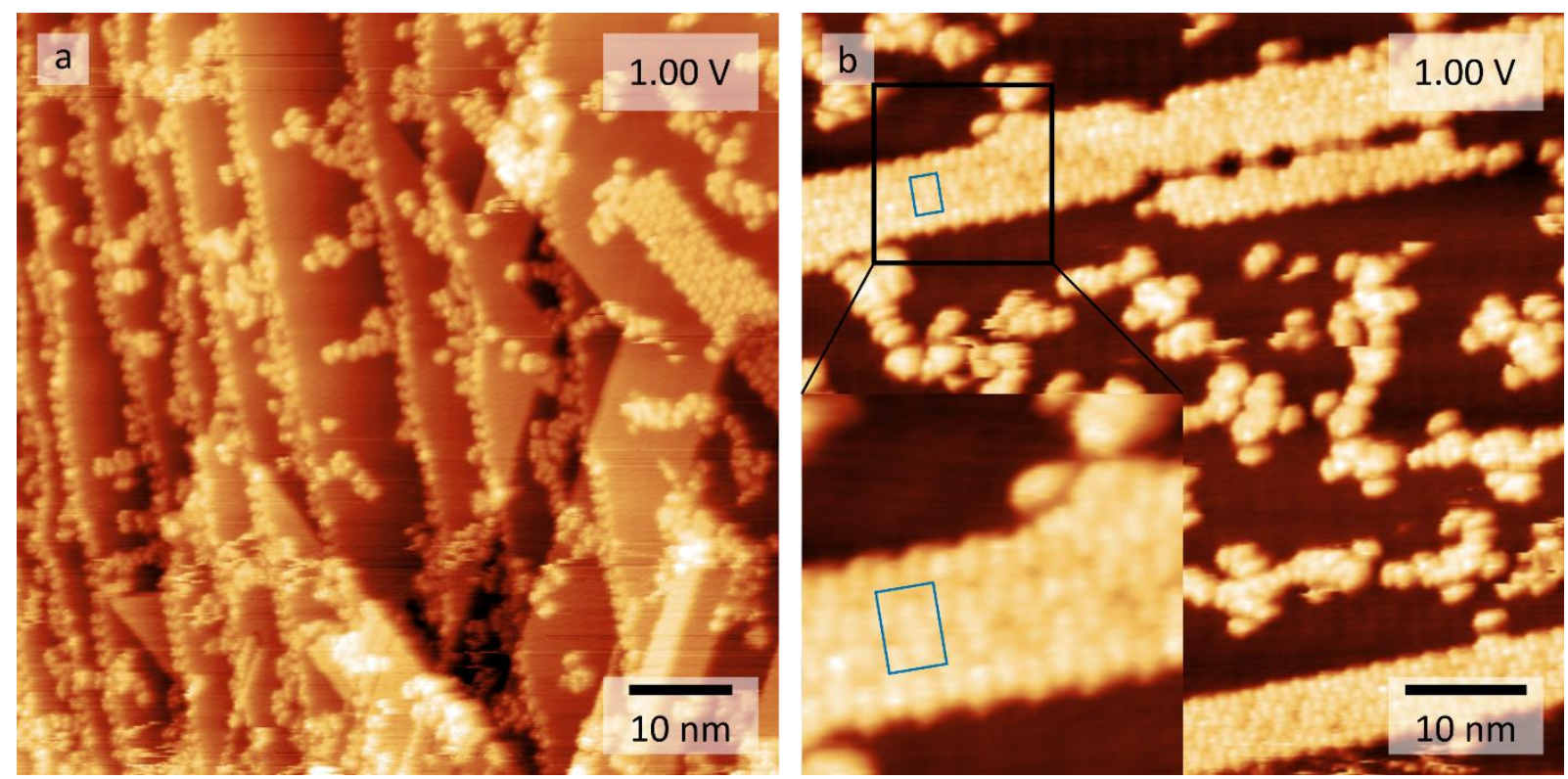

Figure S12: (a, b) STM images of an H-Mabiq submonolayer coverage deposited at $300 \mathrm{~K}$. Panel (a) shows that the H-Mabiq molecules at low coverages grow preferentially at the step edges of the Ag(111) substrate, before forming molecular islands ( $a, b)$. Panel (b) highlights that such islands exhibit the same unit cell, and thus the same local order, as the RT phase of H-Mabiq at saturation coverage (cf. for example the inset in the left-bottom corner with Figure S5). STM measurement parameters: (a) $U_{B}=1.00 \mathrm{~V}, I=0.10 \mathrm{nA} ; 74 \times 74 \mathrm{~nm}^{2}$ (b) $U_{B}=1.00 \mathrm{~V}, I=0.30$ $n A ; 63 \times 63 \mathrm{~nm}^{2}$. 


\section{Observation of Ag-bridged trimetallic Mabiq dimers in solution}

In the course of studies to generate Mabiq coordination compounds-in this case an attempt to generate [Ni(Mabiq)] $\mathrm{PF}_{6}$ upon reaction of [Ni(Mabiq)] with $\mathrm{AgPF}_{6}-$ a trimetallic $\mathrm{Ni}_{2} \mathrm{Ag}-\mathrm{Mabiq}$ product was isolated from the resultant reaction mixture. The silver and nickel containing complex was obtained upon slow evaporation of a concentrated solution of the reaction product in DCM, affording orange crystals suitable for $\mathrm{X}$-ray analysis.

The crystallographic data were collected on an X-ray single crystal diffractometer, equipped with a CCD detector (Bruker APEX II, $\mathrm{k}-\mathrm{CCD}$ ), a rotating anode (Bruker AXS, FR591) with Mo Ka radiation ( $\lambda=$ $0.71073 \AA$ ) and MONTEL mirror optics by using the APEX3 software package ${ }^{1}$. The crystal was picked from perfluorinated ether and fixed on top of a Mitegen ${ }^{\circledR}$ microsampler and transferred to the diffractometer. The measurement was carried out under a constant stream of cold nitrogen. A matrix scan was used to determine the initial unit cell parameters. SAINT ${ }^{2}$ as implemented in the APEX 3 suite was used to merge and correct the reflections for Lorenz and polarization effects, scan speed, and background. Absorption corrections containing odd and even ordered spherical harmonics were performed using SADABS ${ }^{3}$. Space group assignments were established using systematic absences, $\mathrm{E}$ statistics, and successful refinement of the structures. Structures were solved using the intrinsic phasing method (SHELXT), which also supported the correct assignment of the space groups, and were refined against all data using SHELXLE in conjunction with SHELXL-2014 ${ }^{4-6}$. Hydrogen atoms were calculated to the ideal position and refined employing a riding model with isotropic thermal parameters. Non-hydrogen atoms were refined with anisotropic displacement parameters. The images of the crystal structure were generated using Mercury ${ }^{7}$. Employing Platon ${ }^{8}$ Squeeze, 174 electrons in a solvent accessible void of $606 \AA^{3}$ were determined corresponding to 2.5 hexane molecules (175 electrons).

The precise oxidation states of the metals could not be determined from the crystal structure as the data did not allow for a distinction between phosphate, hydrogenphosphate, dihydrogenphosphate and phosphoric acid counterions in the structure. However, due to the diamagnetic nature of the complex, as evidenced by NMR, the oxidation states of the metals are tentatively assigned as +2 for the two nickel ions, and +1 for the silver ion.

CCDC 2095454 contains the supplementary crystallographic data for this compound. These data can be obtained free of charge via www.ccdc.cam.ac.uk/data_request/cif, or by emailing data_request@ccdc.cam.ac.uk, or by contacting The Cambridge Crystallographic Data Centre, 12 Union Road, Cambridge CB2 1EZ, UK; fax: +44 1223336033.

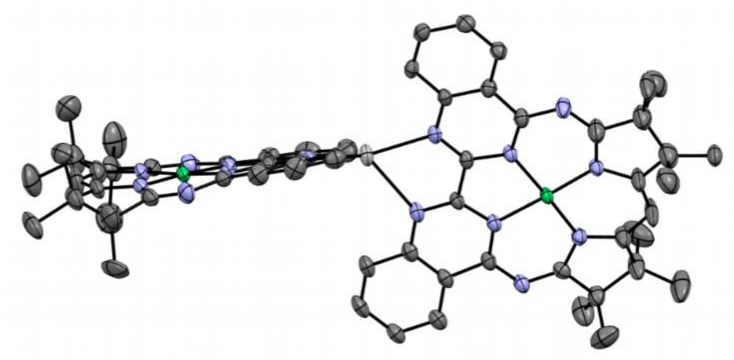

Figure S13: $X$-ray diffraction-based model of the trimetallic Ag-containing Mabiq compound, side view. Ellipsoids are shown with $50 \%$ probability. Counterions have been omitted for clarity. The measured N-Ag distances amount to $2.3 \AA$. The tilt angle between the Ni(Mabiq) molecular planes connected via the bridging Ag atom is found to be $79.1^{\circ}$. 


\section{References}

1. Bruker, APEX Suite of Crystallographic Software, APEX 3. APEX Suite of Crystallographic Software, APEX 3, Version 2015-5.2. 2015.

2. SAINT, Version 8.34A. Bruker AXS Inc.: Madison, Wisconsin, USA 2014; SAINT, Version 8.38A. Bruker AXS Inc.: Madison, Wisconsin, USA 2015.

3. SADABS, Version 2014/5. Bruker AXS Inc.: Madison, Wisconsin, USA 2014.

4. Sheldrick, G., Crystal Structure Refinement with SHELXL. Acta Crystallogr. C 2015, 71, 3-8.

5. Sheldrick, G., SHELXT - Integrated Space-Group and Crystal-Structure Determination. Acta Crystallogr. A 2015, 71, 3-8.

6. Hubschle, C. B.; Sheldrick, G. M.; Dittrich, B., ShelXle: A Qt Graphical User Interface for SHELXL. J. Appl. Crystallogr. 2011, 44, 1281-1284.

7. Macrae, C. F.; Bruno, I. J.; Chisholm, J. A.; Edgington, P. R.; McCabe, P.; Pidcock, E.; Rodriguez-Monge, L.; Taylor, R.; van de Streek, J.; Wood, P. A., Mercury CSD 2.0 - New Features for the Visualization and Investigation of Crystal Structures. J. Appl. Crystallogr. 2008, 41, 466-470.

8. Spek, A., Structure Validation in Chemical Crystallography. Acta Crystallogr. D 2009, 65, 148-155. 